\title{
Consumo selectivo de las marcas periodísticas en España: confianza, orientación política y grado de consumo digital
}

\section{Selective consumption of news brands in Spain: trust, political leaning, and degree of digital consumption}

\author{
Elsa Moreno-Moreno; Elena-Luisa Sanjurjo-San-Martín
}

Cómo citar este artículo:

Moreno-Moreno, Elsa; Sanjurjo-San-Martín, Elena-Luisa (2020). “Consumo selectivo de las marcas periodísticas en España: confianza, orientación política y grado de consumo digital”. Profesional de la información, v. 29, n. 5, e290524.

https://doi.org/10.3145/epi.2020.sep.24

Artículo recibido el 25-06-2020

Aceptación definitiva: 02-10-2020

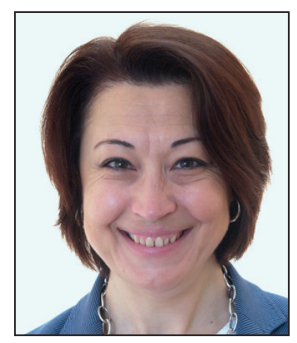

Elsa Moreno-Moreno $\triangle$ https://orcid.org/0000-0003-4066-6475

Universidad de Navarra

Facultad de Comunicación

Departamento de Proyectos Periodísticos Campus Universitario, s/n. Edif. Biblioteca 31009 Pamplona, España emoreno@unav.es

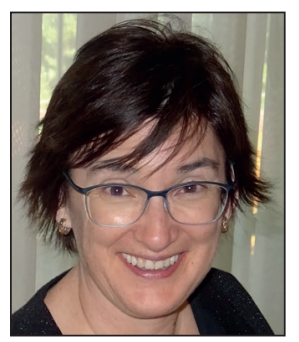

Elena-Luisa Sanjurjo-San-Martín https://orcid.org/0000-0002-9058-0883

Universidad de Navarra

Facultad de Ciencias Económicas y

Empresariales

Campus Universitario, $\mathrm{s} / \mathrm{n}$

31009 Pamplona, España

esanjurjo@unav.es

\section{Resumen}

En el marco de las teorías de la confianza periodística y la exposición selectiva a los medios, se analiza si la confianza que los usuarios de noticias digitales en España declaran profesar a una marca periodística es o no un factor explicativo directo del consumo de dicha marca. Además, y desde una asociación novedosa en la bibliografía, se comprueba si la orientación política y el grado de consumo digital son dos atributos individuales predictores con un efecto directo sobre el consumo. También, se prueba de qué modo la confianza media en este proceso. Esta investigación aplica la metodología del análisis de mediación simple o análisis de efectos directos o indirectos sobre los datos de la encuesta online Digital news report.es 2019 ( $\mathrm{N}=2005)$. Las 15 cabeceras informativas estudiadas son TVE, Antena 3, La sexta, Telecinco, Cuatro, Cadena SER, COPE, El país, El mundo, ABC, La vanguardia, El periódico, 20 minutos, Eldiario.es y El confidencial. Como principal resultado, se identifican dos modelos de consumo selectivo de marcas periodísticas en España. Un primer modelo que contempla un efecto mediador para uno o los dos atributos (tipo I) y un segundo tipo que combina tanto los efectos directos como de mediación o indirectos (tipo II). Esta categorización pone de relieve cómo la orientación política del usuario modera o potencia el consumo de algunas marcas periodísticas al mediar la confianza. A excepción de COPE, los usuarios que se ubican políticamente hacia la derecha confían menos en la marca y, por tanto, el consumo se modera. Por su parte, los usuarios que se sitúan políticamente en la izquierda realizan un consumo más fiel de los medios en los que más confían (Eldiario.es, La sexta y Cadena SER).

\section{Palabras clave}

Noticias; Marcas; Medios; Confianza; Orientación política; Pluralismo informativo; Consumo digital; Exposición selectiva; Usuarios digitales; TVE; Antena 3; La sexta; Telecinco; Cuatro; Cadena SER; COPE; El país; El mundo; ABC; La vanguardia; El periódico; 20 minutos; Eldiario.es; El confidencial.

\section{Financiación}

Este artículo es un resultado del proyecto DigiNativeMedia-Cibermedios nativos digitales en España: caracterización y tendencias (ref. RTI2018-093346-B-C31) del Programa Estatal de I+D+i Orientado a los Retos de la Sociedad, financiado por el Ministerio de Ciencia, Innovación y Universidades de España. 


\begin{abstract}
Within the framework of theories of news trustworthiness and selective exposure to media, this research analyzes whether the trust that consumers of digital news in Spain claim to profess towards a news brand is an explanatory factor that directly mediates their consumption of said brand. In addition, and based on a novel association in literature, this work studies whether the individual attributes of political leaning and degree of digital consumption are predictive variables that have a direct effect on the consumption of news brands. Also, it tests the mediation of trust in this process. This research applies the methodology of simple mediation analysis or analysis of direct or indirect effects to data from the online "Digital news report.es 2019" survey $(\mathrm{N}=2005)$. The 15 news brands studied are TVE, Antena 3, La sexta, Telecinco, Cuatro, Cadena SER, COPE, El país, El mundo, ABC, La vanguardia, El periódico, 20 minutos, Eldiario.es, and El confidencial. As a main result, two models of selective consumption of news brands in Spain are identified: a first model that only considers the mediating effect of one or both attributes (type I), and a second that combines both direct and indirect effects (type II). This categorization highlights how users' political leaning moderates or enhances the consumption of some news brands by mediating trust. With the exception of COPE, users who are politically positioned to the right have less trust in brands, thereby moderating their consumption. On the other hand, users who are politically on the left are more loyal consumers of the media they trust most (Eldiario.es, La sexta, and Cadena SER).
\end{abstract}

\title{
Keywords
}

News; Brands; Media; Trust; Political leaning; Media pluralism; Digital consumption; Selective exposure; Digital users; TVE; Antena 3; La sexta; Telecinco; Cuatro; Cadena SER; COPE; El país; El mundo; ABC; La vanguardia; El periódico; 20 minutos; Eldiario.es; El confidencial.

\section{Introducción}

La confianza que el público declara profesar al periodismo siempre ha sido motivo de interés. Tal y como afirma Fink, "no se trata de un desafío nuevo, pero es persistente y ha ido empeorando" (Fink, 2019, p. 40).

Esta cuestión cobra relevancia ante la revolución crítica de internet, las redes sociales (Samuel-Azran; Hayat, 2019) y las fake news (Bakir; McStay, 2018; Lazer et al., 2018; Tandoc; Jenkins; Craft, 2019). El algoritmo de la Red crece como itinerario informativo y los usuarios digitales demandan a los medios mayor profundidad y vigilancia (Vara-Miguel et al., 2019). Por eso, Lazer et al. (2018, p. 1096) reclaman para el periodismo un "nuevo sistema de salvaguarda" que promueva la verdad. Así se garantizará la calidad de la democracia.

Este contexto sociopolítico calificado por Ladd (2013) como "the era of media distrust" caracteriza a las democracias liberales en las que crece el populismo post-verdad (Speed; Mannion, 2017). Por tanto, la confianza que suscita una marca periodística es un asunto clave. De hecho, los internautas españoles recurren a la marca del medio (54\%) casi tanto como al algoritmo (56\%) para consumir noticias online (Amoedo, 2019). Estudios precedentes muestran que:

- la confianza es una variable explicativa directa del consumo intencional de un medio periodístico (Ladd, 2011; 2013; Stroud; Lee, 2013; Taneja; Yaeger, 2019; Tsfati; Cappella, 2003);

- el público más desconfiado recurre en mayor medida al consumo digital de las noticias (Ardèvol-Abreu; Hooker; Gil de Zúñiga, 2018; Carr et al., 2014; Fletcher; Park, 2017; Ladd, 2011; 2013; Tsfati, 2010; Vara-Miguel, 2020; 2018);

- se observan diferencias en la confianza de la marca según las orientaciones políticas de los usuarios (Fletcher, 2019; Kalogeropoulos, 2018).

No obstante, existe debate en la bibliografía ya que en este proceso intervienen otros factores individuales y contextuales (Fletcher, 2019; Hanitzsch; Van-Dalen; Steindl, 2018; Lee, 2010; Ognyanova, 2019; Tsfati; Ariely, 2014). En este sentido, Vara-Miguel (2019) explica que la tensión política y social reciente en España ha contribuido al descrédito de los medios, cuya credibilidad ha descendido 8 puntos en el último trienio. Este autor señala que sólo un $43 \%$ de los internautas españoles confía habitualmente en las noticias frente a un $31 \%$ de escépticos. Asimismo, es pertinente señalar la influencia del consumo incidental de las noticias a través de los buscadores y las redes sociales (Fletcher; Nielsen, 2018).

Como puede apreciarse, es necesario profundizar en cómo la confianza del público impacta en las variaciones de consumo de los medios (Livio; Cohen, 2018) ya que la confianza es el dilema del periodismo (Lewis, 2019).

El objetivo principal de esta investigación es analizar si la confianza que los usuarios de noticias digitales en España declaran tener en una marca periodística es o no una variable de explicación directa del consumo de dicha marca. Como objetivos secundarios este trabajo comprobará conjuntamente si la orientación política y el grado de consumo digital del público son dos atributos individuales predictores que tienen un efecto directo sobre el consumo. Además, se probará de qué modo la confianza media en este proceso. Una asociación original que ha sido poco estudiada. Sin embargo, la confianza periodística dispone de una larga trayectoria en la bibliografía (véase Kiou-

Ante contextos sociopolíticos diversos, polarizados y complejos, es necesario profundizar en cómo la confianza, la orientación política y el grado de consumo digital del público impactan en el consumo de los medios periodísticos 
sis, 2001; Kohring; Matthes, 2007; Prochazka; Schweiger, 2019; Taneja; Yaeger, 2019; Tsfati; Ariely, 2014; Vara-Miguel, 2018; entre otros, para revisiones) a partir de los trabajos sobre credibilidad (Gaziano; McGrath, 1986; Meyer, 1988). Este artículo aporta la novedad de comprobar si la confianza ejerce efectos de auto-reforzamiento (Ardèvol-Abreu; Hooker; Gil de Zúñiga, 2018) en el consumo de la marca periodística al relacionar confianza, orientación política y grado de consumo digital; o, en cambio, lo debilita o modera.

Para cumplir con los objetivos expuestos, se aplica la metodología del análisis de mediación simple o análisis de efectos directos o indirectos a partir de los datos de la encuesta online Digital news report.es 2019 ( $N=2.005)$. En esta encuesta online se pregunta a los usuarios españoles por la confianza declarada en 15 cabeceras de la actualidad general que se corresponden con los medios informativos principales en términos de audiencia (alcance semanal offline-online entre internautas adultos) de los grupos de comunicación RTVE, Atresmedia, Mediaset, Prisa, Radio Popular-COPE, Unidad Editorial, Vocento, Grupo Godó, Grupo Zeta-Prensa Ibérica, Grupo Henneo, Diario de Prensa Digital S.L. y Titania Compañía Editorial, S.L.

\section{Marco teórico}

\subsection{Confianza y consumo de los medios periodísticos}

Existe debate sobre la asociación positiva entre el grado de confianza que el público declara tener en los medios y su consumo al considerarlos fuentes de noticias creíbles. Algunos estudios identifican asociaciones modestas o marginales (Ardèvol-Abreu; Hooker; Gil de Zúñiga, 2018; Fletcher; Park, 2017; Kiousis, 2001; Tsfati, 2010; Tsfati; Cappella, 2003; 2005; Vara-Miguel, 2018). Sin embargo, otros trabajos reflejan un efecto positivo de la confianza en el consumo de los medios periodísticos (Ladd, 2011; 2013; Stroud; Lee, 2013; Taneja; Yaeger, 2019; Williams, 2012). Asimismo, se advierte que la confianza en la marca informativa difiere por modelo y entidad de medio. Mientras en el Reino Unido, Alemania, Dinamarca, Italia y Japón, el organismo público de radiodifusión es el tipo de marca más

Al relacionar el consumo de medios periodísticos con la confianza, se perciben ciertos patrones de exposición

fiable, la televisión pública española (TVE) está entre las de menos confianza (Kalogeropoulos, 2018, p. 42). También, es significativo que el público exprese más confianza al valorar marcas periodísticas concretas frente a la evaluación genérica de los medios (Daniller et al., 2017), en especial, si se trata de las marcas preferidas para estar informados (Vara-Miguel, 2018).

Al relacionar el consumo de medios periodísticos con la confianza, se perciben ciertos patrones de exposición. Los medios tradicionales como la televisión nacional, la televisión local y los periódicos suscitan más confianza que las noticias online (Tsfati, 2010; Tsfati; Cappella, 2003; Vara-Miguel, 2018; Zhou; Zhang; Shen, 2014). Por tanto, las marcas informativas tradicionales pueden tener una ventaja sobre las marcas digitales, aunque con frecuencia estas últimas producen periodismo de alta calidad y accesible (Kalogeropoulos, 2018, p. 44). Además, Vara-Miguel demuestra en su estudio sobre las similitudes y las diferencias entre las audiencias de los medios tradicionales y los medios nativos digitales de Reino Unido, Alemania, Francia, España e Italia que

"el escepticismo de los medios de comunicación está más extendido entre los usuarios nativos" (Vara-Miguel, 2020, p. 23).

Esto explica que el público desconfiado diversifique sus fuentes informativas hacia los medios nativos digitales y las noticias online (Ardèvol-Abreu; Hooker; Gil de Zúñiga, 2018; Carr et al., 2014; Fletcher; Park, 2017; Ladd, 2011; 2013; Tsfati, 2010; Vara-Miguel, 2020; 2018). Así, los usuarios con bajos niveles de confianza escogen las redes sociales, los blogs y las noticias digitales e interactúan más a través de las formas de participación online (Fletcher; Park, 2017). Algo que contribuye a que los procesos sociales de la Red o digital news sharing otorguen confianza. Por ejemplo, la hipertextualidad y el enlace social favorecen que las noticias compartidas obtengan credibilidad frente a las fuentes tradicionales (Samuel-Azran; Hayat, 2019).

Sobre las razones por las que el público consume medios en los que no confía, Vara-Miguel estima que es debido a que "la gratificación o valor obtenido sería superior al coste de la desconfianza percibida" (Vara-Miguel, 2018, p. 99).

Además, Fletcher y Park recuerdan que

“la confianza y la desconfianza no son nociones antónimas" (Fletcher; Park, 2017, p. 1283).

Estos razonamientos explican el empleo de un medio sólo por su utilidad informativa o disponibilidad de acceso, así como por la gratificación que brinda al público para participar en la discusión social.

\subsection{Orientación política y exposición selectiva}

La teoría de la exposición selectiva o selective exposure se fundamenta en la idea de que las personas seleccionan a propósito mensajes que coinciden con sus creencias (Stroud, 2018). Existe discusión en torno a esta suposición (véase D’Alessio; Allen, 2002; Humanes, 2019; Stroud, 2018; entre otros, para revisiones). D’Alessio y Allen (2002) confirman un apoyo moderado para esta teoría $(r=0,22, p<, 001)$ en su metanálisis de 1956 a 1996. En cambio, otros autores 
certifican que algunos públicos escogen medios percibidos como fuentes periodísticas equilibradas (Bakshy; Messing; Adamic, 2015; Garrett; Stroud, 2014; Metzger; Hartsell; Flanagin, 2015; Powell; Van-der-Meer; Brenes-Peralta, 2019), algo que cuestiona el planteamiento de "echo-chambers" o "burbujas ideológicas" generalizadas (Nelson; Webster, 2017; Zuiderveen-Borgesius et al., 2016). También, la imagen de una ciudadanía polarizada (Nelson; Webster, 2017; Stroud, 2010).

El consumo digital de noticias reaviva la pregunta de cómo son las espirales creadas entre la exposición selectiva a los medios y la inclinación ideológica (Dahlgren; Shehata; Strömbäck, 2019), en ocasiones, según un comportamiento partidista (Mothes; Ohme, 2019; Stroud, 2018; 2010). Por tanto, preocupan los efectos perjudiciales del partidismo en los medios en un momento en el que esta teoría atraviesa una tercera fase de desarrollo que se define por las noticias hiperpartidistas y la diversificación de las conexiones sociales (Barnidge; Peacock, 2019). El interés más personal en las noticias (constatado entre los años 1960 y 2000) supera el paradigma de los "efectos mínimos" (años 1950 y 1960) (Barnidge; Peacock, 2019). Además, Kalogeropoulos (2018) revela cómo la confianza en las marcas periodísticas está influenciada por opiniones preexistentes sobre la política. Este autor indica que Estados Unidos tiene algunos de los públicos de noticias más polarizados del mundo frente a Dinamarca que es el país menos polarizado (Kalogeropoulos, 2018, p. 42). En particular, los públicos de Estados Unidos y Europa con actitudes populistas prefieren la televisión comercial para consumir noticias frente a las noticias online a pesar de que redes sociales como Facebook también sobresalen en este tipo de consumo (Fletcher, 2019).

Por tanto, el tratamiento selectivo de la información (Camaj, 2019) despierta críticas sobre el sesgo partidista de los medios y los sitios web con una agenda política o ideológica partidista (Newman; Kalogeropoulos, 2018). En esta línea, Kim y Choi (2017) comprueban que el consumo diario de internet predice negativamente la confianza en los medios no partidistas y modera la interacción entre las noticias políticas y el debate político. Sin embargo, Dahlgren, Shehata y Strömbäck (2019) señalan que un grado significativo de exposición informativa transversal online obstaculiza el refuerzo entre la exposición selectiva a los medios tradicionales y digitales, y la inclinación ideológica. Por su parte, Ardèvol-Abreu y Gil de Zúñiga (2017) aclaran que el sesgo mediático percibido se asocia con un consumo menor de las noticias en general.

El consumo digital de noticias reaviva la pregunta de cómo son las espirales creadas entre la exposición selectiva a los medios y la orientación política de los usuarios

Estas consideraciones justifican que la orientación política, que es un predictor de la confianza en los medios periodísticos (Ognyanova, 2019), adquiera relevancia como atributo individual del público para determinar de qué modo influye en el consumo selectivo de los mismos.

\section{Hipótesis y metodología}

Presuponiendo que el público consumirá más aquellos medios periodísticos en los que confía (Ladd, 2011; 2013; Stroud; Lee, 2013; Taneja; Yaeger, 2019; Williams, 2012); y que la confianza de los usuarios en las marcas informativas está influenciada por orientaciones políticas preexistentes (Kalogeropoulos, 2018), se parte de las tres hipótesis siguientes:

Hipótesis 1: La confianza, la orientación política y el grado de consumo digital presentan una relación directa sobre el consumo de la marca periodística.

Hipótesis 2: La confianza actúa como factor mediador para la orientación política en el consumo de la marca periodística.

Hipótesis 3: La orientación política declarada por el usuario digital tiene un efecto directo o indirecto (mediado por la confianza), que disminuye o potencia el consumo de la marca periodística.

\subsection{Población y muestra}

La investigación se basa en los datos de la encuesta online de YouGov para el informe Digital news report.es 2019 realizada entre el 17 de enero y el 21 de febrero de 2019 a usuarios de noticias digitales en España. Este informe forma parte del informe global Digital news report que es promovido por el Reuters Institute de la University of Oxford, cuyo socio en España es la Universidad de Navarra. La encuesta online global de YouGov se realizó en 38 países en 2019 con muestras nacionales en torno a los 2.000 usuarios y fue respondida exclusivamente online. En España, con una población de 46 millones, para el año 2019, la muestra tuvo un tamaño $\mathrm{N}=2005$, una penetración de internet del $87 \%$ y un 2,18 de margen de error. El panel de participantes para Research Now SSI (actual Dynata) lo conformaron internautas adultos que consumieron noticias en el último mes, seleccionados según cuotas representativas a nivel nacional para edad, género, nivel de ingresos económicos, nivel educativo y región. Los datos también se ponderaron en función de las metas basadas en datos censales/aceptados por la industria.

A partir del cuestionario de la encuesta online que atiende a 14 preguntas principales, se parte de un análisis exploratorio de los datos sobre varias preguntas de interés para este trabajo. Por un lado, se utilizó de forma directa la pregunta sobre orientación política profesada por el encuestado (Q1F); y la pregunta sobre la confianza declarada en cada una de las 15 marcas evaluadas (Q6 2019 trust grid). De este modo se obtuvieron las variables de estudio de la ideología política 
y la confianza en las marcas periodísticas. Por otro lado, y a partir de las preguntas que permitían al encuestado elegir las marcas que con mayor frecuencia usa para la búsqueda de noticias (Q5_Pregunta única en España desde la 1 hasta la 117), se obtuvo la variable de estudio del grado de consumo digital (GCD) y el porcentaje de consumo de marcas para leer noticias.

En particular, y respecto del grado de consumo, se cuantificó si el número de veces elegido el formato "online" en cualquier marca era mayor, menor o igual al elegido en formato "offline" para obtener tres tipos de clasificación: "online", "híbrido" y "offline". De tal manera que, si la suma de medios en formato "offline" era mayor que la "online", se clasificó el perfil del que responde como "offline" (a la inversa fue "online") y si son parecidas como "híbrido". Finalmente, el consumo de una marca para acceso a noticias se cuantificó a partir de los agregados de la misma sin distinción del formato "online" y "offline". Cada porcentaje fue calculado sobre el total de marcas elegidas para acceso a noticias.

La tabla 1 expone los descriptivos generales para la muestra en relación con las variables de estudio (confianza, orientación política y grado de consumo digital). Esta tabla también incluye la descripción agregada del nivel de confianza en términos generales para las noticias que se eligen y consumen; y las marcas que se consumen.

En referencia a la selección de las marcas periodísticas o medios informativos "online" y "offline" a evaluar, se empleó la muestra de 15 cabeceras de la actualidad general que están presentes en el Digital news report. es 2019 y por las cuales se pregunta al encuestado sobre el grado de confianza que deposita en ellas. Se trata de los medios informativos principales en términos de audiencia (alcance semanal offline-online entre internautas adultos) de los grupos de comunicación primordiales del sector público y privado. El listado corresponde a:

- 5 medios de matriz audiovisual: TVE de RTVE; Antena 3 de Atresmedia; La sexta de Atresmedia; Telecinco de Mediaset y Cuatro de Mediaset;

- 2 medios de matriz radiofónica: Cadena SER de Prisa y COPE del Grupo Radio Popular-COPE;

- 6 medios de matriz impresa: El país de Prisa; El mundo de Unidad Editorial; $A B C$ de Vocento; La vanguardia del Grupo Godó; El periódico del Grupo Zeta-Prensa Ibérica y 20 minutos del Grupo Henneo;

- 2 medios nativos digitales: Eldiario.es de Diario de Prensa Digital S.L. y El confidencial de Titania Compañía Editorial, S.L.
Tabla 1. Descriptivos de las preguntas 6,1 y 5 .

Nivel de confianza general de los usuarios digitales para noticias que se eligen y consumen, orientación política, y grado de consumo digital.

\begin{tabular}{|l|c|c|}
\hline Variables & $\mathbf{N}=\mathbf{2 0 0 5}$ & Porcentaje \\
\hline Nivel de confianza general en noticias: & & \\
Muy en desacuerdo [1] & 216 & 10,8 \\
Tiendo a estar en desacuerdo [2] & 422 & 21,0 \\
Ni de acuerdo ni en desacuerdo [3] & 502 & 25,0 \\
Tiendo a estar de acuerdo [4] & 719 & 35,9 \\
Muy de acuerdo [5] & 146 & 7,3 \\
\hline Nivel de confianza general & & \\
para las marcas que se consumen: & & \\
Muy en desacuerdo [1] & 133 & 6,6 \\
Tiendo a estar en desacuerdo [2] & 376 & 18,8 \\
Ni de acuerdo ni en desacuerdo [3] & 528 & 26,3 \\
Tiendo a estar de acuerdo [4] & 783 & 39,1 \\
Muy de acuerdo [5] & 185 & 9,2 \\
\hline Orientación política: & & \\
Muy a la izquierda & 50 & 2,5 \\
Izquierda & 503 & 25,1 \\
Centro-izquierda & 335 & 16,7 \\
Centro & 318 & 15,9 \\
Centro-derecha & 246 & 12,3 \\
Derecha & 173 & 8,6 \\
Muy a la derecha & 431 & 2,4 \\
No sabe/No contesta & 553 & 16,5 \\
\hline Grado de consumo digital: & 236 & 11,8 \\
1 [offline] & & \\
2 [híbrido] & & \\
3 [online] & & \\
\hline
\end{tabular}

Al igual que en estudios precedentes (Kalogeropoulos, 2018; Kim; Choi, 2017; Kiousis, 2001; Tsfati; Cappella, 2003; Williams, 2012; Zhou; Zhang; Shen, 2014), esta muestra es representativa de la oferta existente en el mercado examinado. En este caso, se trata de medios líderes y distinguidos en el sector periodístico español según Kantar Media para televisión, EGM para radio, OJD para prensa y ComScore para prensa digital. De los diarios regionales y locales, se incluye El periódico por su peso en el ámbito periodístico nacional junto con La vanguardia.

\subsection{Análisis estadísticos empleados}

El análisis elegido para contrastar de forma conjunta las tres hipótesis planteadas fue el de mediación simple o análisis de efectos directos o indirectos, implementada mediante una macro en el programa SPPS ${ }^{1}$. Esta interfaz permite analizar el efecto de una variable mediadora en la relación entre una variable independiente o explicativa y otra dependiente o explicada.

Con anterioridad al análisis de mediación, se emplearon pruebas chi cuadrado entre la confianza y el consumo de cada marca periodística para analizar la independencia de ambas variables. Asimismo, se realizaron cuatro pruebas de tipo no paramétrico o prueba $\mathrm{H}$ de Kruskal-Wallis (extensión de Mann-Whitney a más de dos grupos): una entre el consumo y la orientación política, y otra entre el consumo de la marca y el grado de consumo digital (GCD). Estas pruebas H de Kruskal-Wallis se replicaron de igual manera para la confianza de cada una de las 15 marcas evaluadas. Esta batería de tests 
pretendía asegurar las dos primeras hipótesis. La primera hipótesis referente a si la confianza, la orientación política y el grado de consumo digital (GCD) presentaban diferencias sobre el consumo de las marcas de forma directa (tabla 2). La segunda hipótesis concerniente a si podría existir un efecto mediador de la confianza para las variables de la orientación política y el grado de consumo digital (GCD) (tabla 3). Los resultados de la confianza en la marca en función del grado de consumo digital (GCD) y, posteriormente, según la orientación política arrojaron mayor variación en esta última variable.

Así que, finalmente, la tercera hipótesis se comprobó mediante un análisis de mediación simple.

\section{Resultados}

\subsection{Confianza como principal factor explicativo directo}

Los resultados del análisis de relación directa (pruebas chi cuadrado y pruebas $\mathrm{H}$ de Kruskal-Wallis) otorgan a la confianza las principales diferencias en cuanto al consumo de las 15 marcas periodísticas examinadas. Por tanto, la confianza es presumiblemente uno de los principales factores explicativos directos que determina que el usuario de noticias digitales en España consuma una determinada cabecera u otra. Por su parte, la orientación política y el grado de consumo digital (GCD) se perciben también como variables explicativas directas a la hora de que el usuario seleccione una marca por otra para consumir noticias (tabla 2). Sin embargo, la orientación política y el GCD actúan con menor intensidad frente a la confianza. Asimismo, el análisis de relación directa comprobó que las variables de la orientación política y el GCD ("offline"; "híbrido"; "online") no guardan relación significativa entre ellas en el consumo. De ahí que, sean variables explicativas que ejercen un efecto independiente sobre el consumo.

Tabla 2. Efecto de la confianza; la orientación política y el grado de consumo digital (GCD) en el consumo de cada marca. Prueba chi cuadrado y prueba $\mathrm{H}$ de Kruskal-Wallis.

\begin{tabular}{|c|c|c|c|c|c|c|c|c|c|c|c|c|c|c|c|}
\hline & $M_{1}$ & $M_{2}$ & $M_{3}$ & $M_{4}$ & $M_{5}$ & $M_{6}$ & $M_{7}$ & $M_{8}$ & $M_{9}$ & $M_{10}$ & $M_{11}$ & $M_{12}$ & $M_{13}$ & $M_{14}$ & $M_{15}$ \\
\hline \multicolumn{16}{|c|}{ Consumo de la marca } \\
\hline Confianza & $178,0^{* *}$ & 118,9 & 132,4 & $251,7^{* *}$ & 118,4 & 130,4 & $94,9^{*}$ & $157,4^{* *}$ & $168,8^{* *}$ & 81,0 & $114,0^{* *}$ & 138,8 & $163,1^{* *}$ & $136,5^{*}$ & $174,7^{* *}$ \\
\hline $\begin{array}{l}\text { Orientación } \\
\text { política }\end{array}$ & 11,9 & 5,7 & $43,9^{* *}$ & 7,6 & 20,0 & $14,6^{*}$ & $26,5^{* *}$ & $53,7^{* *}$ & 7,4 & 1,8 & $58,8^{* *}$ & $22,5^{* *}$ & $16,9^{* *}$ & $16,1^{* *}$ & $59,7^{* *}$ \\
\hline GCD & $7,8^{*}$ & 1,1 & $11,9^{* *}$ & $12,0^{* *}$ & $2,3 * *$ & $6,9^{*}$ & 3,4 & 3,2 & $7,9^{* *}$ & 0,9 & 4,9 & 4,7 & 2,1 & $11,3^{* *}$ & 2,1 \\
\hline
\end{tabular}

*significativo al $5 \%$

$* *$ significativo al $1 \%$.

Con $\mathrm{M}_{1}$ : Antena 3; $\mathrm{M}_{2}:$ TVE; $\mathrm{M}_{3}:$ La sexta; $\mathrm{M}_{4}$ : Telecinco; $\mathrm{M}_{5}$ : Cuatro; $\mathrm{M}_{6}:$ El país; $\mathrm{M}_{7}:$ El mundo; $\mathrm{M}_{8}:$ ABC; $\mathrm{M}_{9}: 20$ minutos; $\mathrm{M}_{10}:$ Cadena SER; $\mathrm{M}_{11}:$ COPE; $\mathrm{M}_{12}$ : El periódico; $\mathrm{M}_{13}$ : La vanguardia; $\mathrm{M}_{14}:$ El confidencial; $\mathrm{M}_{15}$ : Eldiario.es.

Estas evidencias muestrales llevaron a concluir que la confianza, la orientación política y el grado de consumo digital presentan una relación directa sobre el consumo de la marca periodística. Por tanto, el consumo de cada cabecera depende de estas tres variables.

\subsection{Mediación de la confianza}

Para comprobar el factor mediador de la confianza, se realizó un estudio preliminar que consistió en un análisis exploratorio gráfico del grado de confianza según la orientación política del encuestado y del grado de confianza en función del grado de consumo digital. Se apreció que las diferencias de respuesta eran mayores cuando se distinguía la variable de la orientación política frente a la variable del grado de consumo digital.

Como puede verse en el gráfico 1, se observaron valoraciones altas de la confianza en las 15 marcas periodísticas respecto del grado de consumo digital. Las valoraciones son superiores a 6 en una escala de Likert de 0 a 10. Es significativo que el grado de consumo de tipo "híbrido" es el que mejor valoración otorga a la confianza de todas las marcas. En particular, destacan El país (de 7 a 8), Cadena SER (de 7 a 8), TVE (de 7 a 8) y Antena 3 (de 7 a 8 ).

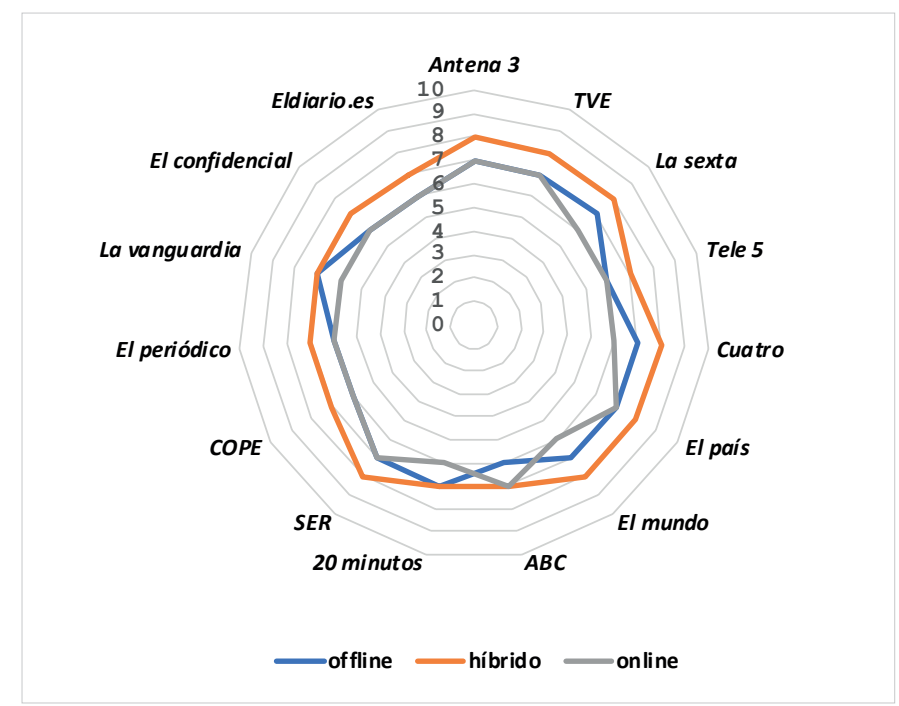

Gráfico 1. Valoración de la confianza según el grado de consumo digital (online-híbrido-offline); de [0] "No confío en absoluto" a [10] "Confío completamente". 
Sin embargo, el gráfico 2 muestra una variación de respuesta mayor para cada una de las 15 marcas informativas en función de la orientación política del usuario. Como puede notarse, los casos de $A B C$ (de 5 a 9), COPE (de 5 a 8) y Antena 3 (de 6 a 8) obtienen una valoración de la confianza más positiva entre los usuarios que se sitúan políticamente en el centro derecha. Por su parte, los casos de El periódico (de 6 a 7), La sexta (de 6 a 8) y Cadena SER (de 6 a 8) despiertan más confianza entre los encuestados que afirman ser de centro izquierda. En concreto, La sexta es la mejor valorada entre el público de izquierda.

Para confirmar los resultados del análisis exploratorio gráfico, se realizaron los tests de Kruskal-Wallis para cada una de las 15 marcas estimadas.
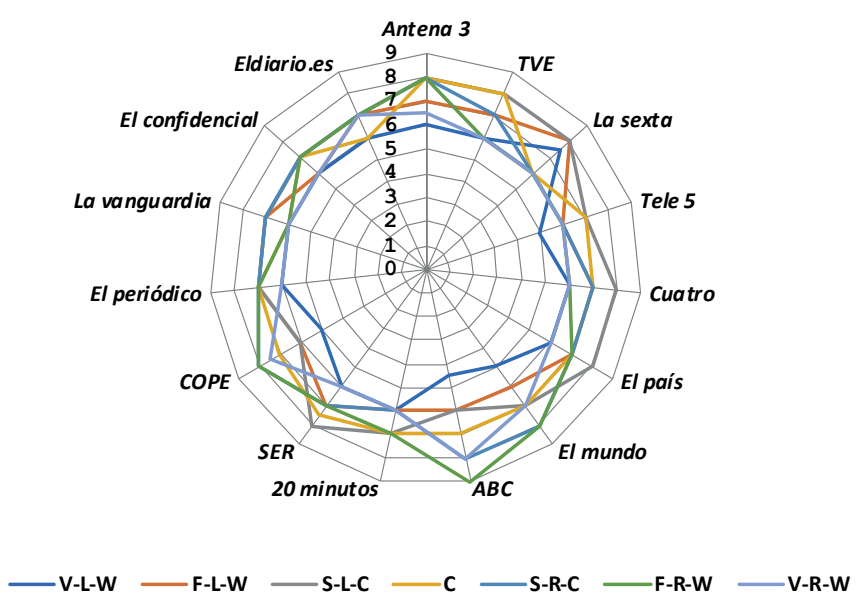

Gráfico 2. Valoración de la confianza según la orientación política del usuario; de [0] “No confío en absoluto" a [10] "Confío completamente".

V-L-W: muy a la izquierda; F-L-W: izquierda; S-L-C: centro-izquierda; C: centro; S-R-C: centro-derecha; F-R-W: derecha; V-R-W: muy a la derecha

Tabla 3. Efecto de la orientación política y el grado de consumo digital (GCD) en la confianza según marca. Prueba H de Kruskal-Wallis.

\begin{tabular}{|l|c|c|c|c|c|c|c|c|c|c|c|c|c|c|c|}
\hline & $\mathbf{M}_{1}$ & $\mathbf{M}_{2}$ & $\mathbf{M}_{\mathbf{3}}$ & $\mathbf{M}_{\mathbf{4}}$ & $\mathbf{M}_{\mathbf{5}}$ & $\mathbf{M}_{\mathbf{6}}$ & $\mathbf{M}_{\mathbf{7}}$ & $\mathbf{M}_{\mathbf{8}}$ & $\mathbf{M}_{\mathbf{9}}$ & $\mathbf{M}_{10}$ & $\mathbf{M}_{11}$ & $\mathbf{M}_{12}$ & $\mathbf{M}_{13}$ & $\mathbf{M}_{14}$ & $\mathbf{M}_{15}$ \\
\hline \multicolumn{10}{|c|}{ Confianza en la marca } \\
\hline $\begin{array}{l}\text { Orientación } \\
\text { política }\end{array}$ & $48,9^{* *}$ & $20,5^{* *}$ & $115,5^{* *}$ & $31,3^{* *}$ & $35,1^{* *}$ & $34,8^{* *}$ & $97,6^{* *}$ & $177,0^{* *}$ & $17,2^{*}$ & $34,0^{* *}$ & $180,7^{* *}$ & $18,7^{*}$ & $13,6^{*}$ & $12,3^{*}$ & $7,7^{*}$ \\
\hline GCD & $35,3^{* *}$ & $19,5^{* *}$ & $20,3^{* *}$ & $43,3^{* *}$ & $34,1^{* *}$ & $14,9^{*}$ & $13,4^{*}$ & $12,3^{*}$ & $19,8^{* *}$ & $24,7^{* *}$ & $13,9^{*}$ & $12,0^{*}$ & $9,2^{*}$ & $12,1^{*}$ & $13,2^{*}$ \\
\hline
\end{tabular}

*significativo al $5 \%$;

$* *$ significativo al $1 \%$.

Con $\mathrm{M}_{1}$ : Antena 3; $\mathrm{M}_{2}:$ TVE; $\mathrm{M}_{3}:$ La sexta; $\mathrm{M}_{4}:$ Telecinco; $\mathrm{M}_{5}:$ Cuatro; $\mathrm{M}_{6}:$ El país; $\mathrm{M}_{7}:$ El mundo; $\mathrm{M}_{8}: A B C ; \mathrm{M}_{9}: 20$ minutos; $\mathrm{M}_{10}:$ Cadena SER; $\mathrm{M}_{11}$ : COPE; $\mathrm{M}_{12}:$ El periódico; $\mathrm{M}_{13}$ : La vanguardia; $\mathrm{M}_{14}:$ El confidencial; $\mathrm{M}_{15}$ : Eldiario.es.

Una vez comprobado que la confianza en la marca periodística difiere más por la orientación política del usuario frente al grado de consumo digital, se optó por analizar la mediación de la confianza únicamente en ella. De este modo, el estudio del grado de consumo digital quedó excluido respecto a su posible efecto indirecto sobre el consumo.

\subsection{Efecto indirecto de la orientación política}

El análisis de mediación simple consideró una combinación de los efectos directos entre el consumo de las marcas periodísticas y las tres variables estudiadas (confianza, orientación política y grado de consumo digital). Este análisis tuvo en cuenta que el único efecto indirecto de la confianza es para la orientación política del usuario. Por tanto, el modelo de mediación simple planteado es el siguiente:

$$
\text { Cons }_{i}=\mathrm{b}_{o}+\mathrm{b}_{1} \times \text { Conf }_{._{i}}+\mathrm{b}_{2} \Varangle \text { G.C.D. } D_{i}+\mathrm{b}_{3} \times \text { Ideolog.Polit } ._{i} \times+\mathrm{b}_{4} \times\left(\text { Conf. }{ }^{*} \text { Ideolog. polit }\right)_{i}+\mathrm{e}_{i}
$$

Para las marcas periodísticas $\mathrm{i}=1 ; 2 ; \ldots . .15$

Los resultados reafirman que en un $80 \%$ de las marcas periodísticas evaluadas la confianza es el principal factor directo explicativo del consumo. Se trata de:

- 4 medios de matriz audiovisual: TVE; Antena 3; La sexta y Telecinco;

- 1 medio de matriz radiofónica: Cadena SER;

- 5 medios de matriz impresa: El país; El mundo; La vanguardia; El periódico; 20 minutos;

- 2 medios nativos digitales: Eldiario.es y El confidencial.

Por su parte, el grado de consumo digital dispone de un impacto explicativo directo menor ya que se prueba únicamente en el caso de 20 minutos. El efecto directo de la orientación política se produce en un $20 \%$ de las marcas (Eldiario.es, La sexta y Cadena SER). Asimismo, se verifica que en un 33,33\% de las cabeceras analizadas la confianza actúa como un factor mediador para la variable de la orientación política. Se corresponde con Eldiario.es, La sexta y la Cadena SER. Además, ni en $A B C$ ni en Cuatro se aprecia el efecto mediador de la confianza y, al no salir significativos los coeficientes del modelo, se han omitido en la tabla 4, en la que pueden comprobarse el resto de las marcas.

La confianza actúa como un factor mediador para la orientación política del usuario al consumir las marcas periodísticas de Eldiario.es, La sexta y Cadena SER 
Tabla 4. Análisis de mediación del consumo de las marcas periodísticas y efecto total (directo+indirecto) sobre el consumo.

\begin{tabular}{|c|c|c|c|c|c|c|c|c|}
\hline & \multicolumn{4}{|c|}{ Efecto directo } & \multirow{2}{*}{$\begin{array}{c}\text { Efecto } \\
\text { indirecto } \\
\text { Conf.* } \\
\text { orientación } \\
\text { política }\end{array}$} & \multicolumn{2}{|c|}{ Validación } & \multirow{2}{*}{$\begin{array}{c}\text { Valoración } \\
\text { del efecto indirecto } \\
\text { sobre el consumo }\end{array}$} \\
\hline & Constante & Confianza & $\begin{array}{l}\text { Orientación } \\
\text { política }\end{array}$ & GCD & & $\mathrm{R}^{2}$ & $\mathrm{~F}$ & \\
\hline$T V E$ & & $0,915^{* *}$ & & & & 0,119 & $7,036^{* *}$ & \\
\hline Antena 3 & & $1,175^{* *}$ & & & & 0,164 & $13,608^{* *}$ & \\
\hline La sexta & $-5,061^{* *}$ & $1,299 * *$ & $0,829^{* *}$ & & $-0,173^{* *}$ & 0,170 & $14,035^{* *}$ & Reduce \\
\hline Tele 5 & & $0,763^{* *}$ & & & & 0,186 & $18,921^{* *}$ & \\
\hline Elmundo & & $0,370^{*}$ & & & & 0,112 & $6,124^{* *}$ & \\
\hline 20 minutos & $-5,846^{*}$ & $1,163^{* *}$ & & $1,167^{*}$ & & 0,152 & $10,805^{* *}$ & \\
\hline SER & $-2,554^{*}$ & $0,635^{* *}$ & $0,476^{*}$ & & $-0,088^{* *}$ & 0,108 & $5,616^{* *}$ & Reduce \\
\hline Elpaís & & $0,828^{* *}$ & & & & 0,125 & $7,781^{* *}$ & \\
\hline COPE & & & & & $0,103^{* *}$ & 0,156 & $11,733^{* *}$ & Aumenta \\
\hline El periódico & $-4,567^{* *}$ & $1,131^{* *}$ & & & $-0,123^{* *}$ & 0,133 & $8,530^{* *}$ & Reduce \\
\hline La vanguardia & & $1,046^{* *}$ & & & & 0,1635 & $12,869 * *$ & \\
\hline El confidencial & & $1,075^{* *}$ & & & & 0,1271 & $7,285^{* *}$ & \\
\hline Eldiario.es & $-8,567^{* *}$ & $2,557^{* *}$ & $1,401^{* *}$ & & $-0,358^{* *}$ & 0,2107 & $21,070^{* *}$ & Reduce \\
\hline
\end{tabular}

Nota: significativo al $5 \%$ ó $* p<.05 ;$ y al $1 \%$ ó **p<.01

Al estudiar el efecto indirecto de la orientación política a través de la mediación de la confianza, se constata que en la mayoría de las marcas periodísticas -a excepción de COPE- cuando se desplaza el espectro de la orientación política de los usuarios hacia posiciones más conservadoras o de derecha, esto provoca una disminución de la confianza en la marca (signo negativo de los coeficientes que miden el efecto indirecto). Por tanto, el consumo de dicha marca se modera.

\section{Discusión y conclusiones}

En el marco de las teorías de la confianza periodística y la exposición selectiva a los medios o selective exposure, esta investigación analiza si la confianza que los usuarios de noticias digitales en España declaran profesar a una marca periodística es o no una variable explicativa de mediación directa en el consumo de dicha marca. Además, y desde una asociación novedosa en la bibliografía, este trabajo demuestra si los atributos individuales de la orientación política y el grado de consumo digital (GCD) del usuario son dos variables predictoras que tienen un efecto directo sobre el consumo de las marcas periodísticas o se trata, más bien, de un efecto mediado a través de la confianza.

De acuerdo al análisis de mediación simple o análisis de efectos directos o indirectos sobre los datos de la encuesta online Digital news report.es 2019 ( $\mathrm{N}=2005)$, se identifican dos modelos de consumo selectivo de marcas periodísticas en España al considerar de manera conjunta y, por un lado, los efectos directos de la confianza, la orientación política y el grado de consumo digital (GCD); y, por otro lado, los efectos indirectos de la orientación política a través de la confianza. Esta investigación aporta a la bibliografía, por tanto, la originalidad de un primer modelo que solo contempla un efecto mediador para uno o los dos atributos (tipo I) y un segundo tipo que combina tanto los efectos directos como de mediación o indirectos (tipo II). Véase gráfico 3.
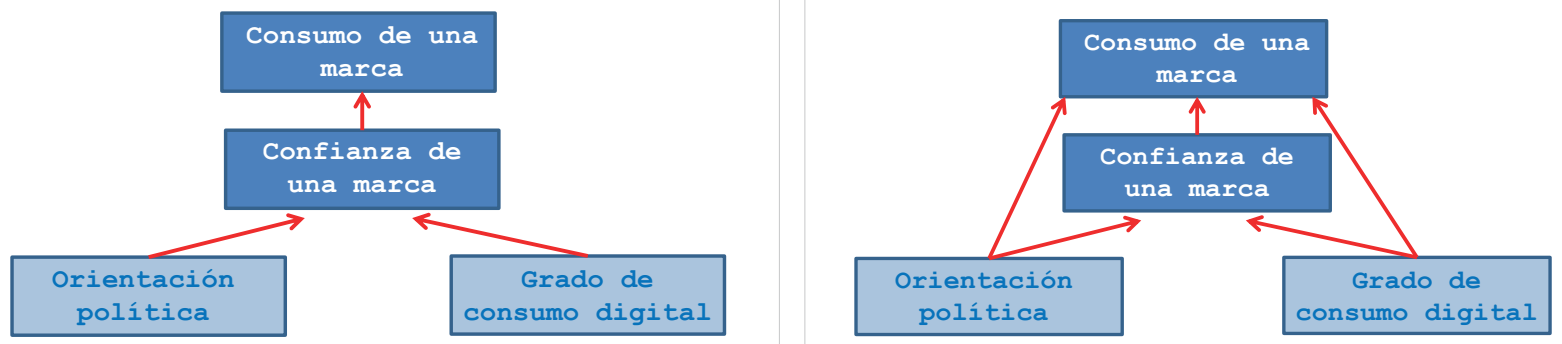

Gráfico 3. Modelos de consumo selectivo de las marcas periodísticas en España. Tipo I (izquierda) y Tipo II (derecha). 
Fruto de esta categorización singular, este trabajo confirma la asociación positiva entre la confianza y el consumo intencional de los medios periodísticos para el caso español al igual que estudios precedentes (Ladd, 2011; 2013; Stroud; Lee, 2013; Taneja; Yaeger, 2019; Williams, 2012). La confianza es el principal factor explicativo directo en el consumo de 12 de las 15 marcas estudiadas (la excepción corresponde a Cuatro, $A B C$ y COPE). Es decir, el $80 \%$ de los casos entre los que están cuatro medios de matriz televisiva, un medio de matriz radiofónica, cinco medios de matriz impresa y dos nativos digitales. En este sentido, y a medida que la valoración de la confianza aumenta, el porcentaje del consumo de una marca concreta crece en relación con el total de las marcas que cada usuario dice emplear. Así, el consumo se incrementa desde un 0,37\% (El mundo) hasta un 2,56\% (Eldiario.es). Por tanto, la hipótesis 1 se valida para la variable explicativa de la confianza, pero esta validación se cumple en menor medida para los atributos individuales de la orientación política y el grado de consumo digital. El efecto directo de la orientación política en el consumo se constata en Eldiario.es, La sexta y Cadena SER (el 20\% de los casos que coinciden con un diario nativo digital, una televisión y una radio). Por su parte, el efecto directo del grado de consumo digital se cumple sólo en 20 minutos; quizá porque este diario dispone de una audiencia sólo online que supera a la audiencia sólo offline y la audiencia dual offline+online. Los comportamientos que acaban de describirse atienden al modelo de consumo selectivo tipificado como de tipo II (gráfico 3).

Asimismo, se confirma la hipótesis 2 parcialmente ya que la confianza actúa como un factor mediador para la orientación política que declara tener el usuario de noticias digitales en España al consumir una marca periodística. Esto se certifica en un 33,33\% de los medios analizados. Los dos modelos de consumo selectivo según efectos directos (tipo I) o mixto (directos e indirectos) (tipo II) ratifican parcialmente esta hipótesis. Así, se advierte el caso con tan sólo un efecto indirecto de la orientación política a través de la confianza (COPE); y ejemplos que atienden al combinado directo e indirecto para la confianza (Eldiario.es, La sexta, Cadena SER y El periódico). De ahí que pueda decirse que este trabajo está en la línea de un apoyo moderado a la teoría de la exposición selectiva a los medios (D’Alessio; Allen, 2002) ya que la confianza de los usuarios españoles en las marcas periodísticas no está totalmente predeterminada por sus orientaciones políticas previas. Sin embargo, se advierte un cierto grado de polarización en la selección de marcas de línea editorial de izquierdas (Eldiario.es, La sexta y Cadena SER). Se trata de un diario nativo digital, una televisión y una radio. Por tanto, el caso español difiere de lo demostrado por Kalogeropoulos (2018) para algunos contextos sociopolíticos que tienen audiencias de noticias más polarizadas como Estados Unidos. Más bien, los usuarios españoles escogen medios en los que confían a modo de fuentes informativas variadas y equilibradas (Bakshy; Messing; Adamic, 2015; Garrett; Stroud, 2014; Metzger; Hartsell; Flanagin, 2015; Powell et al., 2019) sin que la orientación política predetermine la dieta mediática. A diferencia de estudios previos (Mothes; Ohme, 2019; Stroud, 2018; 2010), no se ve un comportamiento partidista por parte de los usuarios de noticias digitales en España.

No obstante, el efecto directo de la orientación política del usuario puede disminuir o potenciarse en aquellas marcas periodísticas sobre las que existe además un efecto mediador indirecto de la confianza sobre el consumo (tabla 4). Esto ocurre en el modelo tipo II, que combina tanto un efecto directo como de mediación y bajo el que se engloban Eldiario.es, La sexta y Cadena

$S E R$. A excepción de COPE, en el resto de los medios se nota que el desplazamiento de la orientación política de los usuarios hacia posiciones más conservadoras o de derecha conlleva que la confianza disminuya en la marca periodística quizá debido a una actitud más crítica del público. Por tanto, el consumo se modera. Por su parte, los usuarios que declaran estar en posiciones ideológicas de izquierda realizan un consumo más fiel de medios en los que confían como Eldiario. es, La sexta y Cadena SER. Esta adhesión vuelve a constatar una cierta polarización en el consumo de marcas de línea editorial de izquierda. Por tanto, la hipótesis 3 también se corrobora.

Finalmente, es pertinente señalar que esta investigación dispone de tres limitaciones:

- La primera limitación se refiere a la población analizada. La muestra de la encuesta online de YouGov para el informe Digital news report.es 2019 presentó un tamaño N=2005 con una población de 46 millones, una penetración de internet del $87 \%$ y un 2,18 de margen de error. El panel de participantes lo conformaron internautas adultos que consumieron noticias en el último mes, seleccionados a través de cuotas representativas a nivel nacional para edad, género, nivel de ingresos económicos, nivel educativo y región. Por tanto, los resultados de este trabajo hay que valorarlos bajo este criterio y no bajo el principio del consumo de marcas periodísticas por parte de la población general en España.

- La segunda limitación afecta a la representatividad de la orientación política declarada por el encuestado la cual puede suponer un cierto sesgo. Al encuestado se le pregunta en qué ubicación política se colocaría. Por tanto, no se le pregunta sobre el recuerdo de voto respecto de las últimas elecciones generales. De ahí que, sea conveniente considerar 
el "voto oculto" en el contexto sociopolítico español. Sin embargo, es pertinente señalar que el encuestado no tiene que posicionarse obligatoriamente en la respuesta sobre la orientación política ya que puede escoger la opción de "No sabe/No contesta" (tabla 1).

- Como tercera limitación, debe tenerse cautela al comparar el caso español con otros contextos sociopolíticos ya que se aprecian grandes variaciones de la confianza en las marcas periodísticas al mediar la orientación política de los usuarios (Kalogeropoulos, 2018). En este sentido, es relevante considerar que factores individuales y contextuales diversos intervienen en este proceso (Fletcher, 2019; Hanitzsch; Van-Dalen; Steindl, 2018; Lee, 2010; Ognyanova, 2019; Tsfati; Ariely, 2014).

Estudios futuros deben profundizar en cómo la orientación política del usuario modera o potencia el consumo del caso específico de los medios nativos digitales al mediar la confianza. Trabajos próximos también podrían ahondar en esta cuestión para el caso concreto de los medios con sesgo partidista y los sitios web con una ideología partidista (Newman; Kalogeropoulos, 2018; Stroud; Lee, 2013; Stroud, 2010; Taneja; Yaeger, 2019). Debido a que la exposición selectiva partidista contribuye a la polarización política (Stroud, 2010), podría comprobarse si la confianza refuerza o modera el consumo de este tipo de medios y sitios web. Por lo tanto, podría obtenerse cuál es el modelo de consumo selectivo para este tipo de marcas. Incluso, valorar si la percepción del sesgo mediático se asocia con un consumo menor de las noticias (Ardèvol-Abreu; Gil de Zúñiga, 2017). Estas investigaciones podrían cruzar variables sociodemográficas para buscar patrones de comportamientos diferentes. Incluso, aplicar estas variables a la relación entre confianza, orientación política y grado de consumo digital. Finalmente, convendría indagar más en cómo se interrelaciona la confianza en las instituciones políticas y la confianza periodística en el caso español. También, en cómo los debates a través de las redes sociales y la comunicación personal intervienen en la confianza de los medios.

En síntesis, es necesario impulsar estudios que arrojen más luz sobre el consumo selectivo de las marcas periodísticas tras demostrar que la orientación política del público modera o potencia el consumo intencional de algunos medios al mediar la confianza. Al igual que los trabajos que inciden en la creación y la medición de credibilidad de los medios (Calvo-Porral; Martínez-Fernández;

Juanatey-Boga, 2014), las aportaciones sobre la confianza ayudarán a que las organizaciones periodísticas enfrenten con certidumbre un contexto sociopolítico diverso, polarizado y complejo. También a que éstas gestionen un mercado híbrido online-offline en el que es conveniente favorecer el consumo intencional de las marcas frente al consumo incidental de las noticias. De este modo, se custodiará la fidelidad del público.

\section{Nota}

1. Descarga gratuita de Process v.3.4 for SPSS by Andrew F. Hayes.

\section{Referencias}

Amoedo, Avelino (2019). "Los algoritmos influyen tanto como la marca de los medios para llegar a las noticias en internet". En: Vara-Miguel, Alfonso; Negredo, Samuel; Amoedo, Avelino; Moreno, Elsa. Digital news report.es 2019. Universidad de Navarra. Center for Internet Studies and Digital Life.

https://www.digitalnewsreport.es/2019/los-algoritmos-influyen-tanto-como-la-marca-de-los-medios-para-llegar-a-lasnoticias-en-internet

Ardèvol-Abreu, Alberto; Gil de Zúñiga, Homero (2017). "Effects of editorial media bias perception and media trust on the use of traditional, citizen, and social media news". Journalism \& mass communication quarterly, v. 94, n. 3, pp. 703724.

https://doi.org/10.1177/1077699016654684

Ardèvol-Abreu, Alberto; Hooker, Catherine M.; Gil de Zúñiga, Homero (2018). “Online news creation, trust in the media, and political participation: Direct and moderating effects over time”. Journalism, v. 19, n. 5, pp. 611-631.

https://doi.org/10.1177/1464884917700447

Bakir, Vian; McStay, Andrew (2018). "Fake news and the economy of emotions: Problems, causes, solutions". Digital journalism, v. 6, n. 2, pp. 154-175.

https://doi.org/10.1080/21670811.2017.1345645

Bakshy, Eytan; Messing, Solomon; Adamic, Lada A. (2015). "Exposure to ideologically diverse news and opinion on Facebook". Science, v. 348, n. 6239, pp. 1130-1132.

https://doi.org/10.1126/science.aaa1160

Barnidge, Matthew; Peacock, Cynthia (2019). "A third wave of selective exposure research? The challenges posed by hyperpartisan news on social media". Media and communication, v. 7, n. 3, pp. 4-7.

https://doi.org/10.17645/mac.v7i3.2257 
Calvo-Porral, Cristina; Martínez-Fernández, Valentín-Alejandro; Juanatey-Boga, Óscar (2014). “Credibilidad de los medios de comunicación: análisis de la prensa diaria desde el comportamiento del consumidor". El profesional de la información, v. 23, n. 3, pp. 300-309.

https://doi.org/10.3145/epi.2014.may.10

Camaj, Lindita (2019). "From selective exposure to selective information processing: A motivated reasoning approach". Media and communication, v. 7, n. 3, pp. 8-11.

https://doi.org/10.17645/mac.v7i3.2289

Carr, D. Jasun; Barnidge, Matthew; Lee, Byung-Gu; Tsang, Stephanie-Jean (2014). "Cynics and skeptics: Evaluating the credibility of mainstream and citizen journalism". Journalism \& mass communication quarterly, v. 91, n. 3, pp. 452-470. https://doi.org/10.1177/1077699014538828

Dahlgren, Peter M.; Shehata, Adam; Strömbäck, Jesper (2019). "Reinforcing spirals at work? Mutual influences between selective news exposure and ideological leaning". European journal of communication, v. 34, n. 2, pp. $159-174$. https://doi.org/10.1177/0267323119830056

D’Alessio, Dave; Allen, Mike (2002). "Selective exposure and dissonance after decisions". Psychological reports, v. 91, n. 2, pp. 527-532.

https://doi.org/10.2466/pr0.2002.91.2.527

Daniller, Andrew; Allen, Douglas; Tallevi, Ashley; Mutz, Diana C. (2017). "Measuring trust in the press in a changing media environment". Communication methods and measures, v. 11, n. 1, pp. 76-85. https://doi.org/10.1080/19312458.2016.1271113

Fink, Katherine (2019). "The biggest challenge facing journalism: A lack of trust". Journalism, v. 20, n. 1, pp. 40-43. https://doi.org/10.1177/1464884918807069

Fletcher, Richard (2019). "The rise of populism and the consequences for news and media use". In: Newman, Nic; Fletcher, Richard; Kalogeropoulos, Antonis; Nielsen, Rasmus-Kleis. The Reuters Institute digital news report 2019. Oxford: Reuters Institute for the Study of Journalism, pp. 42-47. https://reutersinstitute.politics.ox.ac.uk/sites/default/files/2019-06/DNR_2019_FINAL_0.pdf

Fletcher, Richard; Nielsen, Rasmus-Kleis (2018). "Are people incidentally exposed to news on social media? A comparative analysis". New media \& society, v. 20, n. 7, pp. 2450-2468. https://doi.org/10.1177/1461444817724170

Fletcher, Richard; Park, Sora (2017). "The impact of trust in the news media on online news consumption and participation". Digital journalism, v. 5, n. 10, pp. 1281-1299.

https://doi.org/10.1080/21670811.2017.1279979

Garrett, R. Kelly; Stroud, Natalie-Jomini (2014). "Partisan paths to exposure diversity: Differences in pro and counter attitudinal news consumption". Journal of communication, v. 64, n. 4, pp. 680-701. https://doi.org/10.1111/jcom.12105

Gaziano, Cecilie; McGrath, Kristin (1986). "Measuring the concept of credibility". Journalism and mass communication quarterly, v. 63, n. 3, pp. 451-462. https://doi.org/10.1177/107769908606300301

Hanitzsch, Thomas; Van-Dalen, Arjen; Steindl, Nina (2018). "Caught in the nexus: A comparative and longitudinal analysis of public trust in the press". International journal of press/politics, v. 23, n. 1, pp. 3-23.

https://doi.org/10.1177/1940161217740695

Humanes, María-Luisa (2019). "Selective exposure in a changing political and media environment". Media and communication, v. 7, n. 3, pp. 1-3. https://doi.org/10.17645/mac.v7i3.2351

Kalogeropoulos, Antonis (2018). "Which brands do we trust and why?". In: Newman, Nic; Fletcher, Richard; Kalogeropoulos, Antonis; Levy, David A. L.; Nielsen, Rasmus-Kleis. The Reuters Institute digital news report 2018. Oxford: Reuters Institute for the Study of Journalism, pp. 42-43.

http://media.digitalnewsreport.org/wp-content/uploads/2018/06/digital-news-report-2018.pdf

Kim, Hyunjung; Choi, Yoon-jung (2017). "Political discussion, political news, and internet use: Factors of media trust in South Korea". Communication research reports, v. 34, n. 3, pp. 211-220. https://doi.org/10.1080/08824096.2017.1286468

Kiousis, Spiro (2001). "Public trust or mistrust? Perceptions of media credibility in the information age". Mass communication and society, v. 4, n. 4, pp. 381-403.

https://doi.org/10.1207/S15327825MCS0404_4 
Kohring, Matthias; Matthes, Jörg (2007). "Trust in news media: Development and validation of a multidimensional scale". Communication research, v. 34, n. 2, pp. 231-252.

https://doi.org/10.1177/0093650206298071

Ladd, Jonathan M. (2011). Why Americans hate the media and how it matters. Princeton: Princeton University Press. ISBN: 9780691147864

Ladd, Jonathan M. (2013). "The era of media distrust and its consequences for perceptions of political reality". In: Ridout, Travis N. (ed.). New directions in media and politics. London: Routledge, pp. 24-44. ISBN: 9780203109052

Lazer, David M. J.; Baum, Matthew A.; Benkler, Yochai; Berinsky, Adam J.; Greenhill, Kelly M.; Merczer, Filippo; Metzger, Miriam J.; Nyhan, Brendan; Pennycook, Gordon; Rothschild, David; Schudson, Michael; Sloman, Steven A.; Sunstein, Cass R.; Thorson, Emily A.; Watts, Duncan J.; Zittrain, Jonathan L. (2018). "The science of fake news". Insights, v. 359, n. 6380, pp. 1094-1096. https://science.sciencemag.org/content/sci/359/6380/1094.full.pdf

Lee, Tien-Tsung (2010). "Why they don't trust the media: An examination of factors predicting trust". American behavioral scientist, v. 54, n. 1, pp. 8-21.

https://doi.org/10.1177/0002764210376308

Lewis, Seth C. (2019). "Lack of trust in the news media, institutional weakness, and relational journalism as a potential way forward". Journalism, v. 20, n. 1, pp. 44-47.

https://doi.org/10.1177/1464884918808134

Livio, Oren; Cohen, Jonathan (2018). "Fool me once, shame on you: Direct personal experience and media trust". Journalism, v. 19, n. 5, pp. 684-698.

https://doi.org/10.1177/1464884916671331

Metzger, Miriam J.; Hartsell, Ethan H.; Flanagin, Andrew J. (2015). "Cognitive dissonance or credibility? A comparison of two theoretical explanations for selective exposure to partisan news". Communication research, v. 47, n. 1, pp. 3-28. https://journals.sagepub.com/doi/10.1177/0093650215613136

Meyer, Philip (1988). “Defining and measuring credibility of newspapers: developing an index". Journalism \& mass communication quarterly, v. 65, n. 3, pp. 567-574. https://doi.org/10.1177/107769908806500301

Mothes, Cornelia; Ohme, Jakob (2019). "Partisan selective exposure in times of political and technological upheaval: A social media field experiment". Media and communication, v. 7, n. 3, pp. 42-53.

https://doi.org/10.17645/mac.v7i3.2183

Nelson, Jacob L.; Webster, James G. (2017). "The myth of partisan selective exposure: A portrait of the online political news audience". Social media + society, v. 3, n. 3, pp. 1-13.

https://doi.org/10.1177/2056305117729314

Newman, Nic; Kalogeropoulos, Antonis (2018). “Who uses alternative and partisan brands?”. In: Newman, Nic; Fletcher, Richard; Kalogeropoulos, Antonis; Levy, David A. L.; Nielsen, Rasmus-Kleis. The Reuters Institute digital news report 2018. Oxford: Reuters Institute for the Study of Journalism, pp. 45-48.

http://media.digitalnewsreport.org/wp-content/uploads/2018/06/digital-news-report-2018.pdf

Ognyanova, Katherine (2019). "The social context of media trust: A network influence model". Journal of communication, v. 69, n. 5, pp. 539-562.

https://doi.org/10.1093/joc/jqz031

Powell, Thomas E.; Van-der-Meer, Toni G. L. A.; Brenes-Peralta, Carlos (2019). "Picture power? The contribution of visuals and text to partisan selective exposure". Media and communication, v. 7, n. 3, pp. 12-31. https://doi.org/10.17645/mac.v7i3.1991

Prochazka, Fabian; Schweiger, Wolfgang (2019). "How to measure generalized trust in news media? An adaptation and test of scales". Communication methods and measures, v. 13, n. 1, pp. 26-42.

https://doi.org/10.1080/19312458.2018.1506021

Speed, Ewen; Mannion, Russell (2017). "The rise of post-truth populism in pluralist liberal democracies: Challenges for health policy". International journal of health policy and management, v. 6, n. 5, pp. 249-251.

https://doi.org/10.15171/IJHPM.2017.19

Stroud, Natalie-Jomini (2010). “Polarization and partisan selective exposure”. Journal of communication, v. 60, n. 3, pp. 556-576.

https://doi.org/10.1111/j.1460-2466.2010.01497.x 
Stroud, Natalie-Jomini (2018). "Selective exposure theories". In: Kenski, Kate; Jamieson, Kathleen-Hall. The Oxford handbook of political communication.

https://www.oxfordhandbooks.com/view/10.1093/oxfordhb/9780199793471.001.0001/oxfordhb-9780199793471-e-009

Stroud, Natalie-Jomini; Lee, Jae-Kook (2013). "Perceptions of cable news credibility". Mass communication and society, v. 16 , n. 1 , pp. $67-88$. https://doi.org/10.1080/15205436.2011.646449

Tandoc, Edson C.; Jenkins, Joy; Craft, Stephanie (2019). "Fake news as a critical incident in journalism". Journalism practice, v. 13, n. 6, pp. 673-689.

https://doi.org/10.1080/17512786.2018.1562958

Taneja, Harsh; Yaeger, Katie (2019). "Do people consume the news they trust? Incidental news usage and the high-choice media environment". In: Proceedings of the 2019 CHI Conference on human factors in computing systems (CHI 2019). Glasgow, Scotland UK: Association for Computing Machinery.

https://doi.org/10.1145/3290605.3300770

Tsfati, Yariv (2010). "Online news exposure and trust in the mainstream media: Exploring possible associations". American behavioral scientist, v. 54, n. 1, pp. 22-42.

https://doi.org/10.1177/0002764210376309

Tsfati, Yariv; Ariely, Gal (2014). "Individual and contextual correlates of trust in media across 44 countries". Communication research, v. 41, n. 6, pp. 760-782.

https://doi.org/10.1177/0093650213485972

Tsfati, Yariv; Cappella, Joseph N. (2003). "Do people watch what they do not trust? Exploring the association between news media skepticism and exposure". Communication research, v. 30, n. 5, pp. 504-529.

https://doi.org/10.1177/0093650203253371

Tsfati, Yariv; Cappella, Joseph N. (2005). "Why do people watch news they do not trust? The need for cognition as a moderator in the association between news media skepticism and exposure". Media psychology, v. 7, n. 3, pp. $251-271$. https://doi.org/10.1207/S1532785XMEP0703_2

Vara-Miguel, Alfonso (2018). “Confianza en noticias y fragmentación de mercado: el caso español”. Comunicació: Revista de recerca i d'anàlisi, v. 35, n. 1, pp. 95-113.

https://doi.org/10.2436/20.3008.01.168

Vara-Miguel, Alfonso (2019). “Ante una confianza decreciente, la mayoría de los usuarios españoles se previene de la desinformación". En: Vara-Miguel, Alfonso; Negredo, Samuel; Amoedo, Avelino; Moreno, Elsa. Digital news report.es 2019. Pamplona: Universidad de Navarra. Center for Internet Studies and Digital Life.

https://www.digitalnewsreport.es/2019/ante-una-confianza-decreciente-la-mayoria-de-los-usuarios-espanoles-sepreviene-de-la-desinformacion

Vara-Miguel, Alfonso (2020). “Cross-national similarities and differences between legacy and digital-born news media audiences". Media and communication, v. 8, n. 2, pp. 16-27.

https://doi.org/10.17645/mac.v8i2.2733

Vara-Miguel, Alfonso; Negredo, Samuel; Amoedo, Avelino; Moreno, Elsa (2019). “Más y mejor periodismo: Un remedio contra la desinformación". Digital news report.es 2019.

https://www. digitalnewsreport.es/category/2019

Williams, Ann E. (2012). "Trust or bust? Questioning the relationship between media trust and news attention". Journal of broadcasting \& electronic media, v. 56, n. 1, pp. 116-131.

https://doi.org/10.1080/08838151.2011.651186

Zhou, Shuhua; Zhang, Hongzhong; Shen, Bin (2014). "Comparison \& magnitude credibility: Whom to trust when reports are conflicting?". The open communication journal, v. 8, n. 1, pp. 1-8.

https://doi.org/10.2174/1874916X01408010001

Zuiderveen-Borgesius, Frederik J.; Trilling, Damian; Möller, Judith; Bodó, Balázs; De-Vreese, Claes H.; Helberger, Natali (2016). "Should we worry about filter bubbles?". Internet policy review, v. 5, n. 1.

https://doi.org/10.14763/2016.1.401 